Canadian Science Publishing

Canadian Journal of Physiology and Pharmacology Revue canadienne de physiologie et pharmacologie

\title{
Expression of the Frizzled Receptors and their Co-receptors in Calcified Human Aortic Valves
}

\begin{tabular}{|r|l|}
\hline Journal: & Canadian Journal of Physiology and Pharmacology \\
\hline Manuscript ID & cjpp-2017-0577.R1 \\
\hline Manuscript Type: & Article \\
\hline Date Submitted by the Author: & 09-Oct-2017 \\
\hline Complete List of Authors: & $\begin{array}{l}\text { Siddique, Ateeque; McGill University Health Centre, Cardiology and Cardiac } \\
\text { Surgery } \\
\text { Yu, Bin; McGill University Health Centre, Cardiology and Cardiac Surgery } \\
\text { Khan, Kashif; McGill University Health Centre, Experimental Surgery } \\
\text { Buyting, Ryan; McGill University Health Centre, Cardiology and Cardiac } \\
\text { Surgery } \\
\text { Al-Kindi, Hamood ; McGill University Health Centre, Cardiology and Cardiac } \\
\text { Surgery } \\
\text { Alaws, Hossny; McGill University Health Centre, Cardiology and Cardiac } \\
\text { Surgery } \\
\text { Rhéaume, Eric; Montreal Heart Institute } \\
\text { Tardif, Jean-Claude; Montreal Heart Institute } \\
\text { Cecere, Renzo; McGill University Health Centre, Cardiology and Cardiac } \\
\text { Surgery } \\
\text { Schwertani, Adel; McGill University Health Centre, Cardiology and Cardiac } \\
\text { Surgery }\end{array}$ \\
\hline Keyword: & Wnt, LRP, disease, aortic valve stenosis, mRNA \\
\hline Is the invited manuscript for \\
consideration in a Special \\
Issue?:
\end{tabular}




\section{Expression of the Frizzled Receptors and their Co- receptors in Calcified Human Aortic Valves}

Ateeque Siddique, Bin Yu, Kashif Khan, Ryan Buyting, Hamood Al-Kindi, Hossny Alaws, Eric Rheaume**, Jean-Claude Tardif**, Renzo Cecere, Adel Schwertani*

Cardiology, cardiac surgery and pathology, McGill University Health Centre, Montreal, Quebec, Canada.

**Montreal Heart Institute, Montreal, Quebec, Canada

*Address for correspondence: Dr. Adel Schwertani

Cardiology

EM1.2224

Glen, 1001 Décarie Blvd

Montreal, Quebec H4A 3J1

Canada

E-mail: adel.schwertani@mcgill.ca

Phone: 1-514-9341934 ext:43841 


\begin{abstract}
The cellular mechanisms that induce calcific aortic stenosis are yet to be unravelled. Wnt signaling is increasingly being considered as a major player in the disease process. However, the presence of Wnt Frizzled receptors (Fzd) and co-receptors LRP5 and 6 in normal and diseased human aortic valves remains to be elucidated. Immunohistochemistry and qPCR were used to determine Fzd receptor expression in normal and calcified human aortic valve tissue, as well as human aortic valve interstitial cells (HAVICs) isolated from calcified and normal human aortic valves. There was significantly higher mRNA expression of 4 out of the 10 Fzd receptors in calcified aortic valve tissues and 8 out of the 10 in HAVICs, and both LRP5/6 co-receptors in calcified aortic valves $(\mathrm{P}<0.05)$. These results were confirmed by immunohistochemistry, which revealed abundant increase in immunoreactivity for Fzd3, 7 and 8, mainly in areas of lipid core and calcified nodules of diseased aortic valves. The findings of abundant expression of Frizzled and LRP5/6 receptors in diseased aortic valves suggests a potential role for both canonical and noncanonical Wnt signaling in the pathogenesis of human aortic valve calcification. Future investigations aimed at targeting these molecules may provide potential therapies for aortic valve stenosis.
\end{abstract}

Keywords: Wnt, LRP, disease, aortic valve stenosis, mRNA, protein, qPCR, immunohistochemistry.
Abbreviations:
HAVICs = Human Aortic Valve Interstitial Cells;
VICs = Valve Interstitial Cells;
VECs $=$ Valve Endothelial Cells, $\mathrm{AVC}=$ Aortic Valve Calcification; 
EMT $=$ Endothelial-to-Mesenchymal Transition;

$\mathrm{CAV}=$ Calcified Aortic Valve 


\section{Introduction}

Aortic stenosis is the most prevalent valvular heart disease in developed countries, affecting $0.2 \%$ of adults between the ages of 50 and 59 years, and $2.8 \%$ of adults older than 75 years (Otto and Prendergast 2014; Thaden et al. 2014). It is mainly caused by aortic valve calcification (AVC) and is a progressive disease that is currently incurable (Lindman et al. 2013; Maganti et al. 2010). AVC is an active process involving basement membrane disruption, inflammatory and immune cell infiltration, and lipid deposition (Leopold 2012; Li et al. 2013; Merryman and Schoen 2013). Calcific aortic stenosis has been linked to many pathologies, which include the presence of a harsh systolic heart murmur, exercise intolerance, angina, syncope, and heart failure (Das et al. 2000). The aortic valve does not contain any vasculature, and is composed of four cell types: valve endothelial cells (VECs), valve interstitial cells (VICs), smooth muscle cells, and myofibroblasts (Chester and Taylor 2007). VECs function to regulate permeability, thrombogenesis, and cell adhesion, whereas VICs have the ability to contract, secrete cytokines, chemokines, growth factors, matrix components, and remodeling enzymes (Filip et al. 1986). VICs are internally associated with either collagen, glycosaminoglycans, or elastin, and in association form the fibrosa, spongiosa, and ventricularis layers of the aortic valve, respectively (Chen and Simmons 2011; Otto et al. 1994). VICs have been shown to possess the ability to differentiate in both a chondrogenic and osteogenic manner (Chester and Taylor 2007; Mulholland and Gotlieb 1996). Hence, it has been postulated that calcification may arise from the transition of VICs to osteoblast-like bone-forming cells, endothelial-to-mesenchymal transition (EMT) of VECs, and/or matrix vesicle formation resulting in microcalcific nodules (Miller et al. 2010; Piera-Velazquez et al. 2011; Wuthier and Lipscomb 2011). 
Mammalian Wnt signaling comprises 19 wingless (Wnt) ligands, 10 frizzled (Fzd) receptors, and 2 low-density lipoprotein receptor-related protein (LRP) co-receptors (Komiya and Habas 2008). Wnt-Fzd binding leads to the activation of intracellular dishevelled (Dsh/Dvl) phosphoprotein, which then modulates various downstream products, triggering one of three major pathways: the canonical ( $\beta$-catenin-dependent) Wnt pathway, the noncanonical ( $\beta$-catenin-independent) planar cell polarity (PCP) pathway, or the noncanonical $\mathrm{Wnt} / \mathrm{Ca}^{2+}$ pathway (Rao and Kuhl 2010). Wnt signaling has been implicated in heart valve development, specifically regulating EMT and cell proliferation during the initial stages of endocardial cushion and heart valve formation (Flaherty et al. 2012; Tian et al. 2010). Moreover, the canonical Wnt pathway has been shown to play a direct role in AVC (Caira et al. 2006; Gu et al. 2014; Marinou et al. 2012). In active canonical Wnt signaling, Wnt binds Fzd receptors as well as LRP5/6 co-receptors, resulting in the activation of Dsh which inhibits GSK3 activity and disrupts the APC/Axin/GSK3 complex that targets $\beta$-catenin for destruction (Gordon and Nusse 2006; Hatsell et al. 2003; Nusse 2012). This results in $\beta$-catenin stabilization and accumulation in the cytoplasm and subsequently translocation to the nucleus (Fagotto et al. 1998). In the nucleus, $\beta$-catenin associates with members of the TCF/LEF family of transcription factors as well as a number of other factors enabling this complex to act as a transcriptional activator or repressor of Wnt target genes (Brunner 1997; Gordon and Nusse 2006; van de Wetering et al. 1997). The LRP5 Wnt coreceptor regulates bone matrix protein expression in aortic valves and vasculature of mice with hypercholesterolemia and through its interaction with its Wnt ligands, LRP5 activates $\beta$-catenin and is thus an important factor in mediating osteogenesis (Caira et al. 2006). More recently, we have shown an important role for noncanonical Wnts in AVC (Albanese et al. 2017). In the present study, we evaluate for the first time the changes in expression of the Fzd receptors in 
calcified aortic valves (CAVs) through the examination of human biopsy samples using qPCR and immunohistochemical techniques. 


\section{Materials and Methods}

\section{Tissue Collection:}

A total of 17 non-calcified aortic valves and 55 calcified aortic valves were collected during cardiac valve replacement surgeries, along with detailed clinical history (Tables 1 and 2). Tissues were fixed in formalin and embedded in paraffin. Five non-calcified aortic valves (mean age $53 \pm 19 ; 3$ males), and aortic valves with focal calcification $(n=5)$ and severely calcified (advanced) aortic valves $(\mathrm{n}=16)$ were also freshly collected during surgery for valve replacement, snap frozen in liquid nitrogen and stored at $-80^{\circ} \mathrm{C}$ until processed for qPCR. All patients with aortic valve stenosis had severe heart failure (NYHA class III-IV). The study was approved by the ethics committee of the McGill University Health Centre, and informed consent was obtained from all participants involved in this study.

\section{Tissue Expression of Fzd mRNAs:}

Total RNAs were extracted from snap frozen aortic valve tissues using TRIzol (Invitrogen)/RNeasy Mini Kit (Qiagen) combining protocol. Total RNAs $(1 \mu \mathrm{g})$ were used for first strand cDNAs synthesis using SuperScript III First-Strand Synthesis System (Invitrogen). qPCRs were performed using QuantiFast ${ }^{\circledR}$ SYBR Green PCR kit (Qiagen) on LightCycler 1.5 (Roche). The qPCR data was analyzed using PrimePCR Analysis (Bio-RAD), and the relative mRNA expressions of the genes of interest were normalized using the housekeeping gene GAPDH. Sequences of gene-specific primers are listed in Table 3.

\section{Immunohistochemistry}

The paraffin-embedded tissue blocks were cut into $4 \mu \mathrm{m}$ sections using a microtome. The sections were incubated for 1 hour in $10 \%$ normal goat serum/PBS solution, then incubated overnight with the primary antibodies in $0.1 \% \mathrm{BSA} / \mathrm{PBS}$ solution in humid chambers at $4{ }^{\circ} \mathrm{C}$. 
Primary antibodies used were: rabbit anti-human Frizzled 3 (Sigma, SAB4503170 at 1:200), Frizzled 7 (Abcam, ab64636 at 1:75), and Frizzled 8 (Sigma, HPA045025 at 1:50). Secondary biotinylated goat anti-Rabbit IgG (Vector Labs, BA1000 at 1:200) were applied followed by Vectastain ABC complex (Vector Laboratories, Burlingame, CA) according to manufacturer protocol. Immunostaining was visualized by $1 \mathrm{xDAB} / \mathrm{H}_{2} \mathrm{O}_{2}$ solution, subsequently counterstained with hematoxylin, and mounted with Permount (Sigma). Immunostaining without primary antibody or with the primary antiserum preabsorbed with its respective antigen was carried out as negative control. Alizarin Red S and Masson's Trichrome staining protocols were used for calcium and collagen detection, respectively.

\section{Isolation and Culture of HAVICs:}

Primary HAVIC lines were generated from fresh surgically removed human aortic valve leaflets, and cultured in DMEM high glucose medium containing $10 \%$ FBS and $1 \mathrm{x}$ streptomycin/penicillin solution (Invitrogen). Briefly, aortic valve leaflets were washed with 1xHBSS buffer (Invitrogen), cut into small pieces, and then incubated in DMEM media with Collagenase Type II (Sigma, 100U/ml) for 3 hours at $37^{\circ} \mathrm{C}$ in water bath with occasional vortex. The fully digested mixtures were centrifuged at $500 \mathrm{~g}$ at $4{ }^{\circ} \mathrm{C}$ for 10 minutes; the supernatant was transferred into new $50 \mathrm{ml}$ centrifuge tube and centrifuged at $1000 \mathrm{~g}$ at $4{ }^{\circ} \mathrm{C}$ for 10 minutes. Cell pellets were suspended in complete DMEM supplemented with 10\% FBS, 1x antibiotic antimycotic (Invitrogen), and seeded in $75 \mathrm{~cm}^{2}$ culture flasks. HAVICs at passages 3 to 5 were used for experiments.

\section{Statistical Analysis}

Unpaired Student's t-tests were used to assess statistically significant differences in mRNA expression measurements of Fzd receptors between stenotic and non-stenotic HAVICs, normal 
and calcified aortic valve tissue, and bicuspid and tricuspid CAV tissues. The same was used to assess the significance of LRP5/6 expression in normal and calcified bicuspid and tricuspid aortic valve tissue. Statistical analysis was performed using SPSS program Version 15. Data are presented as mean $\pm \mathrm{SD}$. 


\section{Results}

\section{Expression of Fzd and LRP5/6 mRNAs in Normal and Calcified Aortic Valve Tissue}

The mRNA expression of Fzd3, 6, 7, and 8 was significantly increased in calcified human aortic valve tissue, compared to normal human aortic valve tissue (Figure $1 ; \mathrm{P}<0.05$ ). There was no significant difference in mRNA expression of Fzd receptors in bicuspid CAVs compared to tricuspid CAVs (Figure 2). The mRNA expression level of Fzd10 was below the detection limit of the techniques we used, and thus the data has not been included in the study. The mRNA expression of both LRP5 and LRP6 were significantly higher in CAVs (both bicuspid and tricuspid) compared to normal human aortic valves (Figure 3; $\mathrm{P}<0.05$ ). Between bicuspid and tricuspid CAVs, there was no significant difference of the mRNA expression of LRP5 and LRP6 (Figure 3).

\section{Immunohistochemical Analysis of Fzd3, 7 and 8}

There was little-to-no immunoreactivity for Fzd3, 7 and 8 in the normal human aortic valves or normal leaflets of CAVs (Figure 4; A-C). In contrast, there was abundant immunoreactivity for Fzd3 and 7 in the lipid core and calcified nodules of CAVs (Figure 4; D-F). Immunoreactivity for Fzd3 and 7 was also seen in the macrophages and endothelial cells of new microvessels (Figure 4; I-L). There was occasional staining in myofibroblasts as well. There was weak-tomoderate immunoreactivity for Fzd8, mainly in foam cells (lipid core) and in osteoblasts (Figure 4; G-H). Negative control sections for Fzd3, 7 and 8 did not show any immunoreactivity.

\section{Expression of Fzd and LRP mRNAs in Normal and Stenotic HAVICs}

There was a significant upregulation of Fzd1, 2, 3, 5, 6, 7, 8 and 9 mRNA expression in stenotic HAVICs compared to normal (Figure 5; $\mathrm{P}<0.05$ ). Again, the mRNA expression level of Fzd10 was below the detection limit of the techniques we used, and thus has not been included here. 


\section{Discussion}

It is now clear that, unlike the old belief, AVC is an active cellular process rather than gradual calcium deposition due to aging (Mohler et al. 2001). Hence, studies illuminating the role of bone metabolism pathways in AVC such as the Wnt-Fzd system have gained growing interest. Many studies have been performed in both human and animal models in order to evaluate the effect of canonical Wnt pathways in AVC (Caira et al. 2006; Cheng et al. 2003; Rajamannan 2011b). However, studies focusing on the noncanonical Wnt ligands and their downstream effectors remain limited. We have recently reported significantly increased Wnt5a and b, and Wnt11 mRNA levels, as well as increased Wnt5b and Wnt11 protein and immunohistochemical staining levels in calcified human aortic valves when compared to normal human aortic valves. Moreover, incubation of HAVICs with the above 3 noncanonical Wnt ligands led to a significant increase in HAVIC mineralization and induction of other osteogenic mediators such as BMP-2 (Albanese et al. 2017). Here, we demonstrate an increased expression of the Fzd3, 6, 7, and 8 Wnt receptor mRNAs in calcified human aortic valve tissue, compared to normal aortic valves. Also, we demonstrate an increased expression of the LRP5 and LRP6 co-receptor mRNAs in calcified aortic valve tissue, when compared to normal aortic valves. Furthermore, immunohistochemical evaluation has revealed a transition from minuscule immunoreactivity of Fzd3, 7 and 8 in normal aortic valves, to vastly increased immunoreactivity of the same receptors in CAVs. We also demonstrate for the first time that HAVICs obtained from calcified human aortic valves exhibit a significant increase in the mRNA expression in 8 out of the $10 \mathrm{Fzd}$ receptors. The combined results presented here suggest a possible involvement of the Wnt signaling pathway in the pathogenesis of AVC. 
The presence of lipids around calcium deposits along with inflammatory cells (macrophages and $\mathrm{T}$ lymphocytes), angiogenic vessels and increased matrix deposition has been documented in CAVs (Olsson et al. 1999). Valves with higher oxidized LDL (oxLDL) have higher densities of inflammatory cells and a higher concentration of inflammatory mediators. The presence of Fzd receptors in areas of lipid core, inflammatory cells, myofibroblasts, osteoblasts, and HAVICs highly suggests the utilization of these receptors in the Wnt signaling cascade, driving the process of lipid deposition and inflammation toward osteoblastic changes, leading to the calcification of the valve. The upregulation of Fzd receptors in aortic valve tissue and especially in HAVICs is therefore highly likely to contribute to the pathogenesis of AVC. In addition, endothelial cells respond to canonical Wnt signaling in vitro. Noncanonical Wnt5a has been shown to promote capillary-like endothelial network formation and proliferation (Zerlin et al. 2008). This further demonstrates the vital role of Fzd receptors in the pathological angiogenesis seen in AVC.

Calcific atherosclerosis coexists in over $90 \%$ of patients with aortic stenosis, however, calcification is accelerated in the valve compared to regions or aortic and coronary atheroma (Dweck et al. 2013). Like in AVS, the cause of atherosclerosis appears to be related to lipid retention, oxidation, and modification, triggering inflammation at susceptible sites in the walls of major arteries. Both diseases share common risk factors and are initiated by lipid deposition and inflammation, triggered by endothelial damage. The upregulation of Wnt/LRP5 signaling pathway is considered to be a trigger for calcification (Caira et al. 2006; Rajamannan 2011a). LRP5 has been demonstrated to be involved in the innate inflammatory response to lipid infiltration in atherosclerosis, and, on the other hand, LRP5 deficient hypercholesterolemic mice 
display attenuated Wnt signaling and aortic lipid infiltration (Borrell-Pages et al. 2015; BorrellPages et al. 2011). It has also been determined that LRP5/6 is necessary for AVC in hypercholesterolemic mice. Wnt3a stimulates the production of lipid droplets by endocytosed LDL-derived cholesterol (Scott et al. 2015). Thus, the upregulation of LRP5/6 in calcified aortic valves in our present study is indicative of the involvement of the Wnt signaling pathway and the Frizzled LRP5/6 co-receptors in the pathogenesis of AVC and atherosclerosis.

Approximately $64 \%$ of individuals with AVC have bicuspid aortic valves (BAVs), which is the most common congenital heart defect. BAVs are under a significantly higher amount strain compared to tricuspid aortic valves, and increased mechanical stress on VICs contributes to cell injury in AVC (Merryman and Schoen 2013; Szeto et al. 2013). Activation of VICs by shear stress induces inflammatory events and angiogenic activity. Once activated, VICs may then undergo osteoblastic differentiation with bone formation (Lerman et al. 2015). Although insignificant, our results show that expression levels of certain Fzd receptors are higher in calcified BAV tissue. We only employed a limited number of cases in our qPCR analysis therefore, increasing the number of cases studied may lead to significant differences. We previously showed that noncanonical Wnt5b had a significantly higher expression level in calcified BAVs (Albanese et al. 2017). This implies that the Wnt-Fzd pathway may be further upregulated in calcified BAVs.

Unlike the Wnt ligands, which can be categorized as being specific to either the canonical or noncanonical pathway, the Fzd receptors have been shown to be non-specific (Grumolato et al. 2010). This is to say, while the upregulation of Fzd in CAVs is supportive of our previous 
finding that the noncanonical pathway is involved in calcific aortic valve stenosis, it does not discredit the possibility that this upregulation could be due to increased signaling through the canonical Wnt pathway as well. The Wnt co-receptors LRP5 and 6 have been shown to associate with Fzd receptors and aid in the binding of Wnt ligands. The noncanonical Wnt pathway does not utilize these co-receptors, however our current results combined with previous results show a simultaneous increase in noncanonical Wnt ligands and LRP5/6 expression (Rao and Kuhl 2010). A potential explanation for this finding is two-fold: a likely possibility is due to increased canonical signaling in calcified valves, and thus an increased expression of LRP bearing Fzd receptors. Alternatively, it is known that there is significant intracellular crosstalk between the two pathways (Weidinger and Moon 2003).

A comprehensive understanding of the mechanism by which the Wnt pathway leads to AVC may allow researchers to exploit key elements as potential drug targets. Secreted frizzled related proteins (SFRP), dickkopfs (DKK), sclerostin (SOST), and Wnt inhibitory factor-1 (WIF-1) have all been shown to be potent modulators of Wnt signaling (Askevold et al. 2012). Hampson et al. demonstrated a significant negative correlation between circulating levels of Wnt inhibitors (DKK and SOST) and the extent of abdominal aortic calcification, even when age, blood pressure, and cholesterol levels were corrected for (Hampson et al. 2013). Repeated demonstration of the involvement of the Wnt pathway in degenerative aortic valve disease onset and progression vindicates the use of canonical and noncanonical Wnt inhibitors as a potential therapy. Further studies are needed to determine which stage of disease and which step in the pathway inhibition would be most effective. In addition, future studies on the effect of increasing 
Fzd expression in HAVICs will allow us to further understand the role of the Wnt-Fzd pathway in AVC.

In summary, we demonstrate here increased expression of 8 frizzled family receptors, and coreceptors LRP5/6, in the valves of patients with AVC. Given the lack of research examining the role of the Wnt pathway in AVC, these findings contribute to expanding the current literature, and aid in shedding the light for both clinicians and researchers on an important area of research to target components of the Wnt-Fzd pathway as potential pharmacological intervention targets for AVC.

Acknowledgments: None.

Source of Funding: This work was supported by the Canadian Institute of Health Research and NSERC.

Disclosures: None. 


\section{References}

Albanese, I., Yu, B., Al-Kindi, H., Barratt, B., Ott, L., Al-Refai, M., de Varennes, B., Shum-Tim, D., Cerruti, M., Gourgas, O., Rheaume, E., Tardif, J.C., and Schwertani, A. 2017. Role of Noncanonical Wnt Signaling Pathway in Human Aortic Valve Calcification. Arterioscler Thromb Vasc Biol 37(3): 543-552. doi: 10.1161/ATVBAHA.116.308394.

Askevold, E.T., Gullestad, L., Aakhus, S., Ranheim, T., Tonnessen, T., Solberg, O.G., Aukrust, P., and Ueland, T. 2012. Secreted Wnt modulators in symptomatic aortic stenosis. J Am Heart Assoc 1(6): e002261. doi: 10.1161/JAHA.112.002261.

Borrell-Pages, M., Romero, J.C., and Badimon, L. 2015. LRP5 deficiency down-regulates Wnt signalling and promotes aortic lipid infiltration in hypercholesterolaemic mice. J Cell Mol Med 19(4): 770-777. doi: 10.1111/jcmm.12396.

Borrell-Pages, M., Romero, J.C., Juan-Babot, O., and Badimon, L. 2011. Wnt pathway activation, cell migration, and lipid uptake is regulated by low-density lipoprotein receptor-related protein 5 in human macrophages. Eur Heart J 32(22): 2841-2850. doi: 10.1093/eurheartj/ehr062.

Brunner, E.P.O.S.L.B.K. 1997. pangolinencodes a Lef-1 homologue that acts downstream of Armadillo to transduce the Wingless signal in Drosophila. Nature Nature 385(6619): 829-833.

Caira, F.C., Stock, S.R., Gleason, T.G., McGee, E.C., Huang, J., Bonow, R.O., Spelsberg, T.C., McCarthy, P.M., Rahimtoola, S.H., and Rajamannan, N.M. 2006. Human degenerative valve disease is associated with up-regulation of low-density lipoprotein receptor-related protein 5 receptor-mediated bone formation. J Am Coll Cardiol 47(8): 1707-1712. doi: 10.1016/j.jacc.2006.02.040.

Chen, J.H., and Simmons, C.A. 2011. Cell-matrix interactions in the pathobiology of calcific aortic valve disease: critical roles for matricellular, matricrine, and matrix mechanics cues. Circ Res 108(12): 15101524. doi: 10.1161/CIRCRESAHA.110.234237.

Cheng, S.L., Shao, J.S., Charlton-Kachigian, N., Loewy, A.P., and Towler, D.A. 2003. MSX2 promotes osteogenesis and suppresses adipogenic differentiation of multipotent mesenchymal progenitors. J Biol Chem 278(46): 45969-45977. doi: 10.1074/jbc.M306972200.

Chester, A.H., and Taylor, P.M. 2007. Molecular and functional characteristics of heart-valve interstitial cells. Philos Trans R Soc Lond B Biol Sci 362(1484): 1437-1443. doi: 10.1098/rstb.2007.2126.

Das, P., Pocock, C., and Chambers, J. 2000. The patient with a systolic murmur: severe aortic stenosis may be missed during cardiovascular examination. QJM 93(10): 685-688.

Dweck, M.R., Khaw, H.J., Sng, G.K., Luo, E.L., Baird, A., Williams, M.C., Makiello, P., Mirsadraee, S., Joshi, N.V., van Beek, E.J., Boon, N.A., Rudd, J.H., and Newby, D.E. 2013. Aortic stenosis, atherosclerosis, and skeletal bone: is there a common link with calcification and inflammation? Eur Heart J 34(21): 1567-1574. doi: 10.1093/eurheartj/eht034.

Fagotto, F., Gl,ck, U., and Gumbiner, B.M. 1998. Nuclear localization signal-independent and importin/karyopherin-independent nuclear import of $\leq$-catenin. Current biology : CB 8(4): 181-190.

Filip, D.A., Radu, A., and Simionescu, M. 1986. Interstitial cells of the heart valves possess characteristics similar to smooth muscle cells. Circ Res 59(3): 310-320.

Flaherty, M.P., Kamerzell, T.J., and Dawn, B. 2012. Wnt signaling and cardiac differentiation. Prog Mol Biol Transl Sci 111: 153-174. doi: 10.1016/B978-0-12-398459-3.00007-1.

Gordon, M.D., and Nusse, R. 2006. Wnt Signaling: Multiple Pathways, Multiple Receptors, and Multiple

Transcription Factors. Journal of Biological Chemistry 281(32): 22429-22433. doi: 10.1074/jbc.R600015200.

Grumolato, L., Liu, G., Mong, P., Mudbhary, R., Biswas, R., Arroyave, R., Vijayakumar, S., Economides, A.N., and Aaronson, S.A. 2010. Canonical and noncanonical Wnts use a common mechanism to activate completely unrelated coreceptors. Genes Dev 24(22): 2517-2530. doi: 10.1101/gad.1957710. 
Gu, G.J., Chen, T., Zhou, H.M., Sun, K.X., and Li, J. 2014. Role of Wnt/beta-catenin signaling pathway in the mechanism of calcification of aortic valve. J Huazhong Univ Sci Technolog Med Sci 34(1): 33-36. doi: 10.1007/s11596-014-1228-x.

Hampson, G., Edwards, S., Conroy, S., Blake, G.M., Fogelman, I., and Frost, M.L. 2013. The relationship between inhibitors of the Wnt signalling pathway (Dickkopf-1(DKK1) and sclerostin), bone mineral density, vascular calcification and arterial stiffness in post-menopausal women. Bone 56(1): 42-47. doi: 10.1016/j.bone.2013.05.010.

Hatsell, S., Rowlands, T., Hiremath, M., and Cowin, P. 2003. [beta]-catenin and Tcfs in mammary development and cancer. Journal of Mammary Gland Biology and Neoplasia 8(2): 145-158.

Komiya, Y., and Habas, R. 2008. Wnt signal transduction pathways. Organogenesis 4(2): 68-75.

Leopold, J.A. 2012. Cellular mechanisms of aortic valve calcification. Circ Cardiovasc Interv 5(4): 605614. doi: 10.1161/CIRCINTERVENTIONS.112.971028.

Lerman, D.A., Prasad, S., and Alotti, N. 2015. Calcific Aortic Valve Disease: Molecular Mechanisms and Therapeutic Approaches. Eur Cardiol 10(2): 108-112. doi: 10.15420/ecr.2015.10.2.108.

Li, C., Xu, S., and Gotlieb, A.I. 2013. The progression of calcific aortic valve disease through injury, cell dysfunction, and disruptive biologic and physical force feedback loops. Cardiovasc Pathol 22(1): 1-8. doi: 10.1016/j.carpath.2012.06.005.

Lindman, B.R., Bonow, R.O., and Otto, C.M. 2013. Current management of calcific aortic stenosis. Circ Res 113(2): 223-237. doi: 10.1161/CIRCRESAHA.111.300084.

Maganti, K., Rigolin, V.H., Sarano, M.E., and Bonow, R.O. 2010. Valvular heart disease: diagnosis and management. Mayo Clin Proc 85(5): 483-500. doi: 10.4065/mcp.2009.0706.

Marinou, K., Christodoulides, C., Antoniades, C., and Koutsilieris, M. 2012. Wnt signaling in cardiovascular physiology. Trends Endocrinol Metab 23(12): 628-636. doi: 10.1016/j.tem.2012.06.001.

Merryman, W.D., and Schoen, F.J. 2013. Mechanisms of calcification in aortic valve disease: role of mechanokinetics and mechanodynamics. Curr Cardiol Rep 15(5): 355. doi: 10.1007/s11886-013-0355-5.

Miller, J.D., Weiss, R.M., Serrano, K.M., Castaneda, L.E., Brooks, R.M., Zimmerman, K., and Heistad, D.D. 2010. Evidence for active regulation of pro-osteogenic signaling in advanced aortic valve disease. Arterioscler Thromb Vasc Biol 30(12): 2482-2486. doi: 10.1161/ATVBAHA.110.211029.

Mohler, E.R., 3rd, Gannon, F., Reynolds, C., Zimmerman, R., Keane, M.G., and Kaplan, F.S. 2001. Bone formation and inflammation in cardiac valves. Circulation 103(11): 1522-1528.

Mulholland, D.L., and Gotlieb, A.I. 1996. Cell biology of valvular interstitial cells. Can J Cardiol 12(3): 231-236.

Nusse, R. 2012. Wnt Signaling. Cold Spring Harbor Perspectives in Biology 4(5). doi: 10.1101/cshperspect.a011163.

Olsson, M., Thyberg, J., and Nilsson, J. 1999. Presence of oxidized low density lipoprotein in nonrheumatic stenotic aortic valves. Arterioscler Thromb Vasc Biol 19(5): 1218-1222.

Otto, C.M., Kuusisto, J., Reichenbach, D.D., Gown, A.M., and O'Brien, K.D. 1994. Characterization of the early lesion of 'degenerative' valvular aortic stenosis. Histological and immunohistochemical studies. Circulation 90(2): 844-853.

Otto, C.M., and Prendergast, B. 2014. Aortic-valve stenosis--from patients at risk to severe valve obstruction. N Engl J Med 371(8): 744-756. doi: 10.1056/NEJMra1313875.

Piera-Velazquez, S., Li, Z., and Jimenez, S.A. 2011. Role of endothelial-mesenchymal transition (EndoMT) in the pathogenesis of fibrotic disorders. Am J Pathol 179(3): 1074-1080. doi: 10.1016/j.ajpath.2011.06.001.

Rajamannan, N.M. 2011a. The role of Lrp5/6 in cardiac valve disease: experimental hypercholesterolemia in the ApoE-/-/Lrp5-/- mice. J Cell Biochem 112(10): 2987-2991. doi: 10.1002/jcb.23221.

Rajamannan, N.M. 2011b. The role of Lrp5/6 in cardiac valve disease: LDL-density-pressure theory. J Cell Biochem 112(9): 2222-2229. doi: 10.1002/jcb.23182.

Rao, T.P., and Kuhl, M. 2010. An updated overview on Wnt signaling pathways: a prelude for more. Circ Res 106(12): 1798-1806. doi: 10.1161/CIRCRESAHA.110.219840. 
Scott, C.C., Vossio, S., Vacca, F., Snijder, B., Larios, J., Schaad, O., Guex, N., Kuznetsov, D., Martin, O., Chambon, M., Turcatti, G., Pelkmans, L., and Gruenberg, J. 2015. Wnt directs the endosomal flux of LDL-derived cholesterol and lipid droplet homeostasis. EMBO Rep 16(6): 741-752. doi: 10.15252/embr.201540081.

Szeto, K., Pastuszko, P., del Alamo, J.C., Lasheras, J., and Nigam, V. 2013. Bicuspid aortic valves experience increased strain as compared to tricuspid aortic valves. World J Pediatr Congenit Heart Surg 4(4): 362-366. doi: 10.1177/2150135113501901.

Thaden, J.J., Nkomo, V.T., and Enriquez-Sarano, M. 2014. The global burden of aortic stenosis. Prog Cardiovasc Dis 56(6): 565-571. doi: 10.1016/j.pcad.2014.02.006.

Tian, Y., Cohen, E.D., and Morrisey, E.E. 2010. The importance of Wnt signaling in cardiovascular development. Pediatr Cardiol 31(3): 342-348. doi: 10.1007/s00246-009-9606-Z.

van de Wetering, M., Cavallo, R., Dooijes, D., van Beest, M., van Es, J., Loureiro, J., Ypma, A., Hursh, D., Jones, T., Bejsovec, A., Peifer, M., Mortin, M., and Clevers, H. 1997. Armadillo Coactivates Transcription Driven by the Product of the Drosophila Segment Polarity Gene dTCF. Cell 88(6): 789799.

Weidinger, G., and Moon, R.T. 2003. When Wnts antagonize Wnts. J Cell Biol 162(5): 753-755. doi: 10.1083/jcb.200307181.

Wuthier, R.E., and Lipscomb, G.F. 2011. Matrix vesicles: structure, composition, formation and function in calcification. Front Biosci (Landmark Ed) 16: 2812-2902.

Zerlin, M., Julius, M.A., and Kitajewski, J. 2008. Wnt/Frizzled signaling in angiogenesis. Angiogenesis 11(1): 63-69. doi: 10.1007/s10456-008-9095-3. 


\section{Figure Captions:}

\section{Figure 1:}

Comparison of mRNA expression of frizzled receptors in human aortic valve tissue isolated from normal $(n=5)$ and calcified aortic valves $(n=13), * P<0.05$.

\section{Figure 2:}

Comparison of mRNA expression of frizzled receptors in human aortic valve tissue isolated from calcified bicuspid aortic valves $(n=7)$ and calcified tricuspid aortic valves $(n=6)$.

\section{Figure 3:}

Comparison of LRP5/6 mRNA expression in human aortic tissue isolated from normal aortic valves $(n=5)$, calcified bicuspid aortic valves $(n=4)$, calcified tricuspid valves $(n=16), * P<0.05$.

\section{Figure 4:}

Representative images of Fzd immunoreactivity in normal and CAV. Panels A-C show the immunostaining of Fzd3 (A), Fzd7 (B), and Fzd8 (C) in normal human aortic valves. Panels D-F show the abundant immunostaining of Fzd3 (D and E) and Fzd7 (F) in the lipid core (LC) and calcified nodules $(\mathrm{CN})$ of calcified valves. Panel $\mathbf{G}$ and $\mathbf{H}$ show the moderate immunostaining of Fzd8 in the lipid core and osteoblasts (OS) of calcified valves. Panels I-L show the immunoreactivity of Fzd3 ( $\mathbf{I}$ and $\mathbf{J}$ ) and Fzd7 (K and $\mathbf{L}$ ) in the macrophages (MP) and endothelial cells (EC) of new microvessels (MV), as well as in myofibroblasts (MF). Scale bar: $100 \mu \mathrm{m}$.

\section{Figure 5:}

Comparison of mRNA expression of frizzled receptors in cultured human aortic interstitial cells (HAVICs) isolated from non-stenotic $(n=5)$ and stenotic $(n=7)$ aortic valves. Most of the Fzds were upregulated in stenotic HAVICs, ${ }^{*} \mathrm{P}<0.05$. 


\section{Tables}

Table 1: Clinical data of patients with aortic valve disease used in immunohistochemistry.

\begin{tabular}{ll}
\hline Clinical Parameter & Mean \pm SD \\
\hline Age (years) & $67 \pm 13$ \\
Female (n) & $26(36.1 \%)$ \\
HbA1c (\%) & $6.03 \pm 1.34$ \\
Total Cholesterol (mmol/L) & $3.90 \pm 1.09$ \\
LDL (mmol/L) & $2.11 \pm 0.92$ \\
HDL (mmol/L) & $0.98 \pm 0.26$ \\
LDL/HDL & $2.19 \pm 0.73$ \\
Triglycerides (mmol/L) & $1.83 \pm 1.97$ \\
Creatinine $(\mu \mathrm{mol} / \mathrm{L})$ & $84.65 \pm 21.20$ \\
Circulating Calcium (normalized to albumin) & $527.94 \pm 88.26$ \\
Aortic Valve Area (cm $\left.{ }^{2}\right)$ & $1.29 \pm 0.84$ \\
Parsonnet Score & $20.74 \pm 12.94$ \\
Ejection Fraction $(\%)$ & $53 \pm 11$ \\
\% of Patients with Congestive Heart Failure & $63.9 \%$ \\
\% of Patients with Diabetes & $27.8 \%$ \\
\% of Patients with Hypertension & $58.3 \%$ \\
\hline
\end{tabular}

Abbreviations: SD: standard deviation; HbAlc: glycated hemoglobin; LDL: low density lipoprotein; HDL: high density lipoprotein

Table 2: Clinical data of patients with aortic valve disease used in qPCR.

\begin{tabular}{ll}
\hline Clinical Parameter & Mean \pm SD \\
\hline Age (years) & $68 \pm 10$ \\
Female $(\%)$ & $33.3 \%$ \\
HbA1c $(\%)$ & $6.07 \pm 1.12$ \\
Total Cholesterol $(\mathrm{mmol} / \mathrm{L})$ & $4.85 \pm 1.66$ \\
LDL $(\mathrm{mmol} / \mathrm{L})$ & $3.32 \pm 1.55$ \\
HDL $(\mathrm{mmol} / \mathrm{L})$ & $1.60 \pm 0.48$ \\
Triglycerides $(\mathrm{mmol} / \mathrm{L})$ & $1.25 \pm 0.24$ \\
Creatinine $(\mu \mathrm{mol} / \mathrm{L})$ & $88.70 \pm 20.83$ \\
Aortic Valve Area $\left(\mathrm{cm}^{2}\right)$ & $1.60 \pm 0.86$ \\
Ejection Fraction $(\%)$ & $53 \pm 14$ \\
\hline
\end{tabular}

Abbreviations: SD: standard deviation; HbA1c: glycated hemoglobin; LDL: low density lipoprotein; HDL: high density lipoprotein 


\section{Table 3: qPCR primer sequences}

\begin{tabular}{lll}
\hline Gene ID & Forward 5'- & Reverse 5'- \\
\hline FZD1 & GTTACAGGGTGAGTGAGATAACCAA & CAAACCCATACCAGGTTTTAGACAC \\
FZD2 & GCCATCCTATCTCAGCTACAAGTTT & CTCCTCCTGTGAGAAGAACATGGAA \\
FZD3 & TCTGAGGATAGCATTTCTCAAGACC & TCCCAAACCACAGATTCCATCTTAG \\
FZD4 & ATTGTAGCTTCCTCAGAAATCCCTT & GAAAGGAATCTACCAGAAAGGAGGT \\
FZD5 & GAAGCACTTCTTTGTCTCTGTTCTC & TAAGTAGATCCAGTTTCTCTCCCCT \\
FZD6 & AGCAAATTGGCTCCGTTTAAGGATT & CTTTCCTGAAAATGAGTCCTGGGT \\
FZD7 & GCCTGATGTACTTTAAGGAGGAGG & CCAGGTAGGTGAGAACGGTAAAGAG \\
FZD8 & CAAAATTGAGTTTTGCACCTTCCC & TTGCAGAAGTTCCATTTACATCCAC \\
FZD9 & AAAATTTTCATGTCACTGGTGGTGG & CCAGGTCTGGAAAGTCTTGGAG \\
FZD10 & AAGTTACCCAAAGGGCTTATTGACT & CAAATATACACACGCAGAAACCACT \\
GAPDH & TGCACCACCAACTGCTTAGC & GGCATGGACTGTGGTCATGAG \\
\hline
\end{tabular}


Canadian Journal oN Pamsiblogyłhd PharmacologyPage 22 of 26

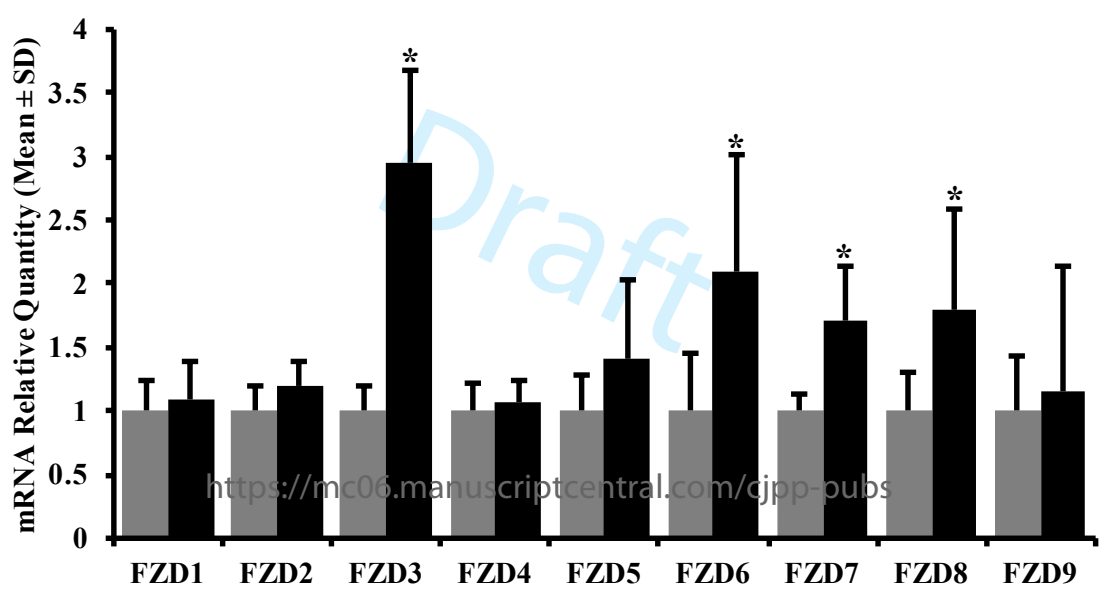




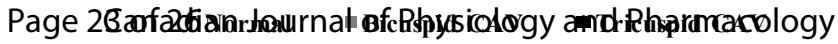
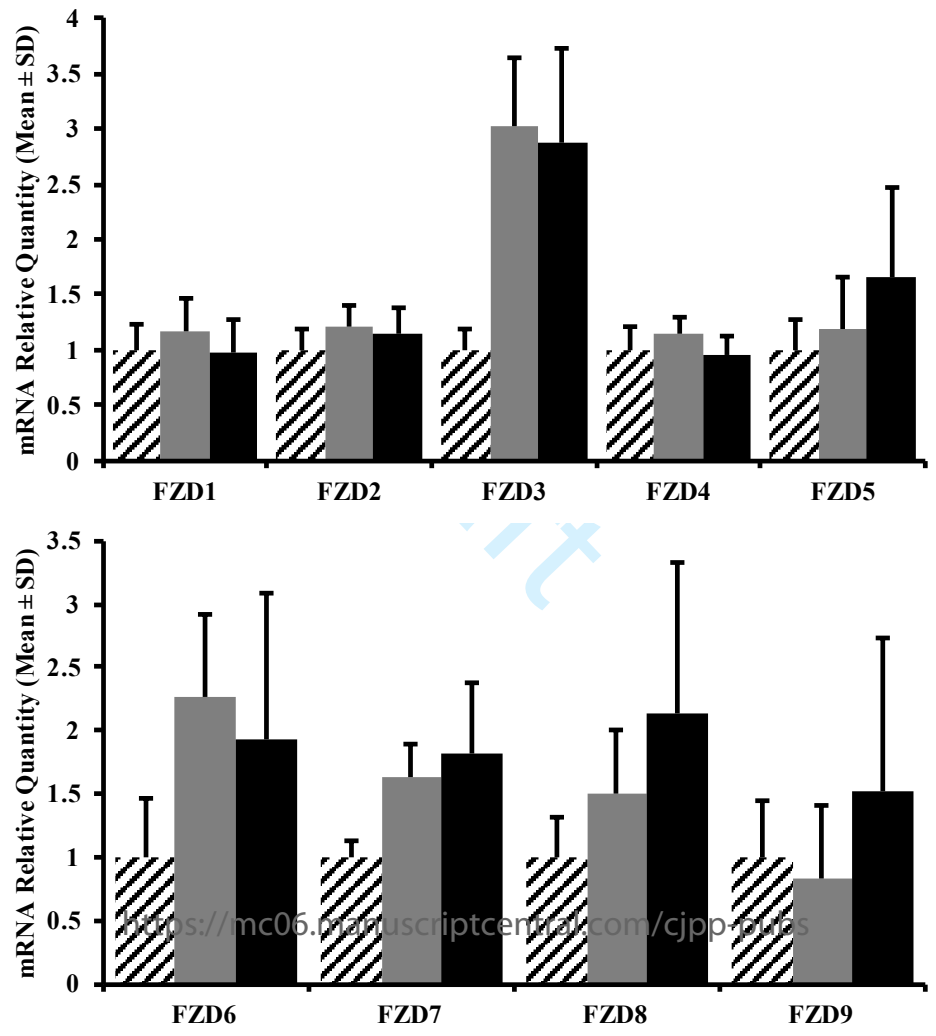


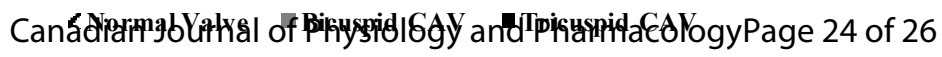

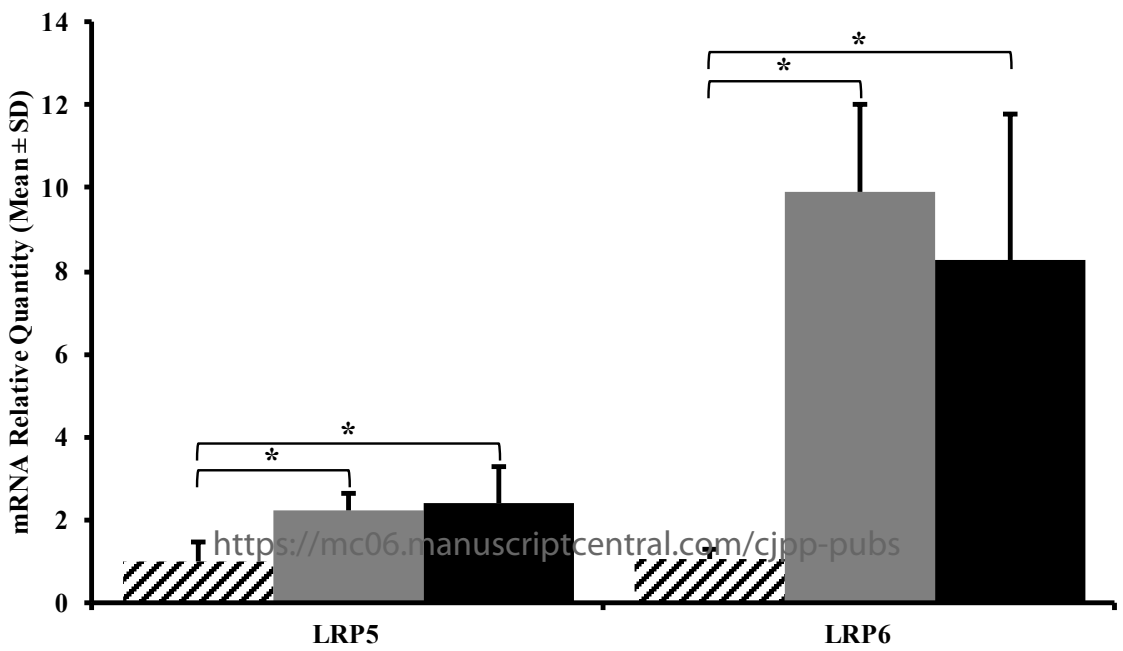




\section{Aage 25anąбan Journal of Physiology and Pharmacology}

$100 \mu \mathrm{m}$

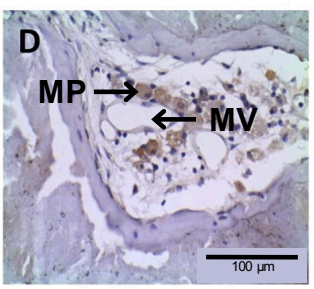

G

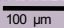

\section{P}

$30 \leftarrow M P$

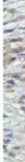

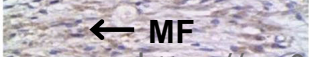

$\mathrm{E}$

F
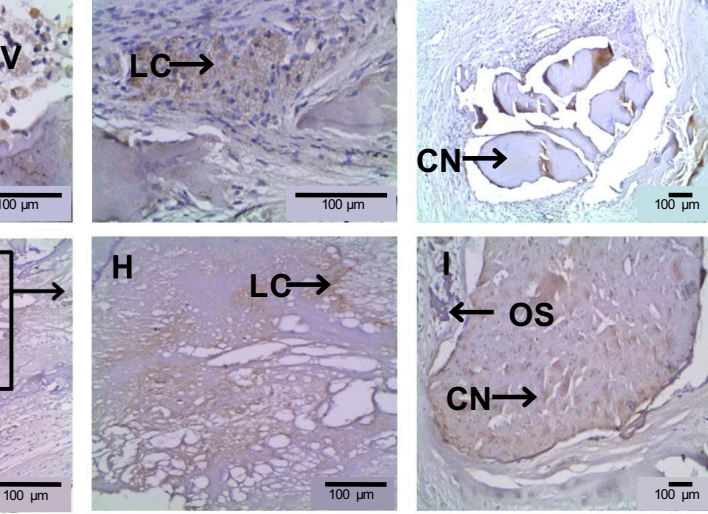

$100 \overline{\mu m}$

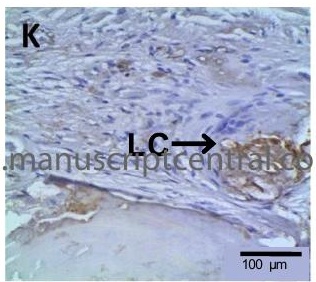

$100 \mu \mathrm{m}$

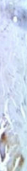


- Non-stenotic HAVICs $\square$ Stenotic HAVICs

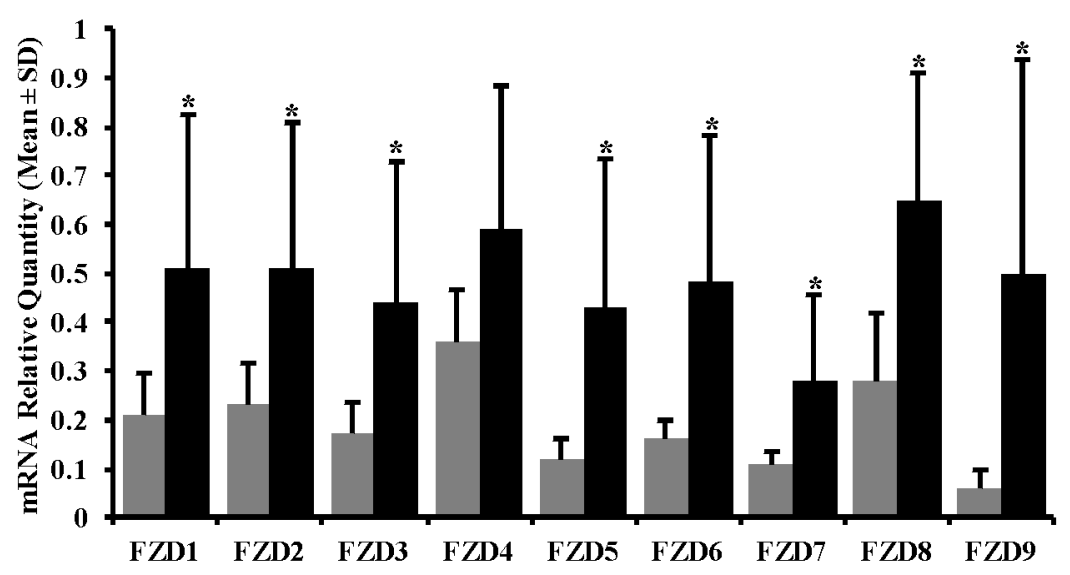

